Journal of research in health science

2018 № 1 (2), Januar-April

www.journalofresearch.org; info@journalofresearch.org

DOI 10.26739/2523-1243

JOURNALL OF RESEARCH

IN HEALTHSCIENCE

ISSN Print: 2523-1243; ISSN Online: 2523-1251

\title{
Cytogenetic manifestations of chromosomal rearrangements in karyotypes of children with attention deficit hyperactivity disorder
}

\section{Saidkhodjaeva S.N.}

Tashkent Pediatric Medical Institute, Tashkent, Republic of Uzbekistan.

Email address:

doctor-saida1986@mail.ru (Saidkhodjaeva S.N.)

To cite this article:

Saidkhodjaeva S.N. Cytogenetic manifestations of chromosomal rearrangements in karyotypes of children with attention deficit hyperactivity disorder. Journal of research in health science. Vol. 1, No. 2, 2018, pp. 23-30. DOI 10.26739/2523-1243

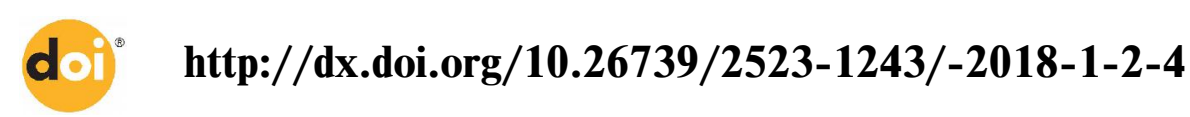

Abstract: In this article, based on a cytogenetic study of 62 children with attention deficit hyperactivity disorder it was found that an increase in the length of the Y-chromosome is associated with the development of ADHD. It is also one of the factors predicting the likelihood of syndrome formation and used in screening and prenatal diagnosis, with the purpose of early detection of predisposition to the development of the syndrome and the adoption of preventive measures.

Keywords: attention deficit hyperactivity disorder, karyotype, Y-chromosome, phenotype, asociality, children.

\section{Цитогенетические проявления хромосомных реаранжировок в кариотипе детей с синдромом дефицита внимания с гиперактивностью}

Резюме: В данной статье на основании цитогенетического исследования 62 детей с синдромом дефицита внимания с гиперактивностью, было установлено, что увеличение длины Y-хромосомы ассоциировано с развитием СДВГ. Также это является одним из факторов прогнозирования вероятности формирования синдрома и использоваться в скрининговой и пренатальной диагностике, с целью раннего выявления предрасположенности к развитию синдрома и принятия профилактических мер.

Ключевые слова: синдром дефицита внимания с гиперактивностью, кариотип, Ү-хромосома, фенотип, асоциальность, дети. 
Saidkhodjaeva S.N. Cytogenetic manifestations of chromosomal rearrangements in karyotypes of children with attention deficit hyperactivity disorder.

\section{Актуальность:}

Проявления особенностей психического развития индивидуальны и во многом зависят от наследственно детерминируемых генетических факторов. Индивидуальная генетическая предрасположенность к определенному развитию нервной системы обусловлена вовлеченностью множества генов. Гены, участвующие в развитии и функционировании нервной системы, в формировании нейрокогнитивного и поведенческого профиля, могут существовать в нескольких аллельных вариантах и встречаться в различных популяциях c разной частотой. Генетический полиморфизм является одной из причин различных фенотипических проявления генов в развитии и функционировании нервной системы и формировании психического статуса.

В ряде случаев отклонения фенотипических проявлений могут быть причиной генетических мутаций. Мутации могут проявляться на геномном, хромосомном и генном уровне и затрагивать различные механизмы реализации генетической программы. Так, мутационные перестройки, приводящие к уменьшению (делеции) или увеличению (амплификации) генетического материала приводят, соответственно, к снижению или увеличению экспрессии отдельных вовлеченных генов. Влияние эффекта "дозы" генов также обуславливает различные изменения фенотипов и варианты их проявлений.

Известно, что в формировании нейрокогнитивного и поведенческого профиля могут участвовать гены, связанные с половыми хромосомами, в том числе - гены NLGN4Y, SRY, PCDH11Y, локализованные на Ухромосоме [Durand Christelle, Kappeler Caroline, 2006; Mulligan A., Gill M., 2008; Ross J.L., Tartaglia N., 2015; Loke H., Harley V., 2015]. Показано, что увеличение числа копий генов Ухромосомы ведет к гиперэкспрессии генных продуктов, участвующих в развитии и функционировании головного мозга. Влияя на формирование нейробиологических основ психического статуса, вовлеченные гены могут служить потенциальным фактором, увеличивающим риск различных изменений поведенческого и социального фенотипа - в частности при наличии эффекта "дозы" генов. Тем не менее, специфические гены, ассоциируемые с поведенческим фенотипом, и молекулярные механизмы когнитивных расстройств, связанных с половыми хромосомами, до конца не изучены [Visootsak J., Graham J.M. Jr., 2009; Cordeiro Lisa, Tartaglia Nicole, 2012].

Цитогенетическим проявлением хромосомных реаранжировок, приводящих к изменению экспрессии вовлеченных генов, является увеличение генетического материала У-хромосомы. Оно может происходить в результате увеличения числа копий У-хромосомы, например, при синдромах, связанных с кариотипами, в которых присутствует одна или более добавочной У-хромосомы: 47, ХУУ [Visootsak J., Graham J.M. Jr., 2009]; 48,XХУУ [Tartaglia N.R., Ayari N., 2012]; 49,ХУУУУ [Paoloni-Giacobino A., Lespinasse J., 2007] и др.

Было показано, что наличие сверхчисленной У-хромосомы у 
Journal of research in health science. 2018; 1 (2): 23-30.

мужчин оказывает влияние на развитие головного мозга, в частности, ассоциировано с увеличением объема белого и серого вещества, обуславливает особенности моторики, речи и поведенческой регуляции [Bryant D.M., Hoeft F., 2012; Lepage J.F., Hong D.S., 2014]. K настоящему времени доказано, что увеличение генетического материала У-хромосомы связано с социальноэмоциональными проблемами, обусловленными такими поведенческими чертами, как нарушение внимания, гиперактивность и импульсивность. В частности, индивидуумы с кариотипами ХУУ и ХХУУ предрасположены к развитию синдрома дефицита внимания и гиперактивности (Attention-deficit hyperactivity disorder, ADHD) [Tartaglia N.R., Ayari N., 2012; Ross J.L., Roeltgen D.P., 2012; Ross J.L., Tartaglia N., 2015]. Это подтверждается еще и тем фактом, что ADHD-синдром более характерен для лиц мужского пола [Mulligan A., Gill M., 2008]. Показано, что ADHD-синдром встречается у 70-82\% мужчин с кариотипом, включающим дополнительную (синдромы 47,ХУУ; 48,ХХУУ), тогда как в общей популяции его частота составляет 3-10\% [Tartaglia N., Davis S., 2006; Visootsak Jeannie , Graham John M. , 2009; Tartaglia Nicole , Ayari Natalie , 2011; Tartaglia Nicole R. , Ayari Natalie, 2012]. Основными фенотипическими проявлениями наличия в геноме дополнительного материала У-хромосомы являются невнимательность, отвлекаемость, низкие организаторские способности, гиперактивность и/или импульсивность; чаще, чем в основной популяции встречаются агрессивность, асоциальность и склонность к насилию. Ранними исследованиями была описана большая встречаемость мужчин с дополнительным материалом У- хромосомы в кариотипе среди заключенных и контингента специальных учреждений для лиц с ментальной ретардацией [Visootsak Jeannie, Graham John M., 2009]. Фенотип, обусловленный дополнительным материалом Ухромосомы, проявляется как результат эффекта "дозы" генов при более высоком уровне их экспрессии по сравнению с таковым при нормальном мужском кариотипе $(46, X У)$ [Tartaglia Nicole, Ayari Natalie, 2011].

Увеличение генетического материала У-хромосомы также может быть следствием либо амплификации, либо встраивания в хромосому более или менее протяженного участка из другой гомологичной или негомологичной хромосомы. Подобное изменение хромосом, цитогенетически проявляемое увеличением общей длины хромосомы, а также длины р- или q-плеча, до настоящего времени считалось полиморфизмом, который встречается в различных популяциях с определенной частотой. При этом в популяционных исследованиях частота выраженных вариантов размеров длинного плеча Ухромосомы составляет около $5,6 \%$. Однако еще в конце XX в исследованиями McConville B.J. и соавт. было показано, что увеличение длины q-плеча $\mathrm{У}$-хромосомы $\left(\mathrm{Yq}^{+}\right)$ ассоциировано с повышенным риском психических расстройств и гиперактивностью. При этом выраженность симптоматики коррелировала со степенью 
Saidkhodjaeva S.N. Cytogenetic manifestations of chromosomal rearrangements in karyotypes of children with attention deficit hyperactivity disorder.

увеличения q-плеча. Подобные проявления могут быть обусловлены влиянием эффекта "дозы" генов, который закономерно возникает при появлении в геноме добавочного хромосомного материала, приводящего к увеличению длины Ухромосомы.

Однако исследования связи размеров У-хромосомы с развитием синдрома дефицита внимания и гиперактивности у детей до настоящего времени не проводились.

Цель исследования: провести анализ ассоциации СДВГ с вариабельностью размера Y-хромосомы, а также с другими возможными цитогенетически детектируемыми изменениями кариотипа.

Материалы и методы исследования: Наиболее информативным методом, позволяющим оценить весь кариотип, является стандартный цитогенетический метод исследования (СЦИ). Данным методом возможно обнаружить не только полную форму цитогенетического отклонения от нормы, но и мозаичную. Это позволяет сделать вывод о происхождении хромосомных изменений (герминальном, эмбриональном) и возможности их передачи потомству. При обследовании детей с СДВГ для хромосомного анализа в качестве биологического образца использовали лимфоциты периферической крови, стимулированные митогеном фитогемаглютинином (ФГА).

Нами было обследовано 62 ребенка в возрасте 6 - 15 лет с клинически диагностированным СДВГ, среди которых было 75,8\% (47) мальчиков и 24,2\% (15) девочек. Девочки были включены в исследование с целью оценки возможной вовлеченности в цитогенетические варианты при СДВГ не только Ү-хромосомы, но и других хромосом - как половых, так и аутосом. Полученные результаты в сравнительном аспекте сопоставлялись с результатами кариотипирования условно здоровых детей - без клинически установленных признаков СДВГ (группа контроля, включавшая 45 детей, в том числе $21(46,7 \%)$ мальчика и 24 (53,3\%) девочки). Ранжировка обследуемых на основании гендерного признака показала, что количество детей с кариотипом 46,ХУ, у которых был диагностирован СДВГ, было в 1,6 раза больше ( $t=2,32 ; \mathrm{p}<0,05)$, чем детей без синдрома, тогда как количество детей с кариотипом 46,XX с признаками СДВГ и без таковых статистически достоверно не различалось $(\mathrm{t}=1,94$; $p>0,05)$. Данный факт дает цитогенетическое основание для подтверждения факта того, что индивидуумы, носящие в кариотипе Y-хромосому, т.е. мальчики, более подвержены развитию СДВГ.

Исследование показало, что среди детей основной группы кариотип без цитогенетически детектируемых изменений половых хромосом встречался в $45,1 \pm 7,2 \%$ случаев у мальчиков и в $100 \%$ случаев у девочек (всего у $69,3 \pm 5,8 \%$ детей с СДВГ). В группе контроля доля метафаз с кариотипом без изменений половых хромосом у мальчиков составила $85,7 \pm 7,6 \%$, а у девочек - $100 \%$ (всего у 93,3 $\pm 3,7 \%$ детей). Сравнение данных основной и контрольной групп в целом показало, что у детей с СДВГ доля кариотипа без цитогенетически детектируемых изменений половых 
хромосом была в 1,3 раза меньше $(\mathrm{t}=3,49 ; \mathrm{p}<0,05)$, чем у детей без клинических признаков синдрома. Отсутствие изменений со стороны половых хромосом у девочек в нашем исследовании может свидетельствовать об отсутствии ассоциации цитогенетических вариаций Х-хромосом с СДВГ. Однако, то, что в нашем исследовании такой зависимости не было обнаружено, может быть и следствием малочисленности девочек, включенных в обследование методом СЦИ. Для уточнения данного факта в дальнейшем необходимо проведение дополнительного исследования с участием большего количества девочек.

Изменения половых хромосом у $54,9 \%$ мальчиков затрагивали в основном длину Y-хромосомы. Данное цитогенетическое проявление до настоящего времени считалось не патологией, а полиморфизмом и расценивалось как вариант нормы. Однако в свете последних данных, касающихся выявления связи СДВГ с увеличением материала Yхромосомы [Tartaglia N.R., Ayari N., 2012; Ross J.L., Roeltgen D.P., 2012; Ross J.L., Tartaglia N., 2015] и влияния эффекта "дозы" генов [Tartaglia Nicole, Ayari Natalie, 2011], необходимо учитывать не только количество Ү-хромосом в кариотипе, но и увеличение размеров данной хромосомы. В нашем исследовании у детей с СДВГ мы не обнаружили кариотипов со сверхчисленными Ү-хромосомами. Однако у 19 из $47(40,4 \pm 7,2 \%)$ обследованных мальчиков с СДВГ было выявлено увеличение размера Y-хромосомы.
Увеличенная Y-хромосома была выявлена и в кариотипе одного мальчика из группы контроля.

Таблица 1. Результаты цитогенетических исследований

\begin{tabular}{|l|c|c|c|c|c|c|}
\hline \multicolumn{1}{|c|}{ Пол } & общий & $\mathrm{Yp}^{+}$ & $\mathrm{Yq}^{+}$ & норма & $\mathrm{XX}$ & $\mathrm{XУ}$ \\
\hline Мальчики & 47 & 1 & 18 & 28 & 1 & 46 \\
\hline Девочки & 15 & - & - & 15 & 15 & - \\
\hline Всего & 62 & 1 & 18 & 27 & 16 & 46 \\
\hline Контроль & 51 & 0 & 1 & 50 & - & 51 \\
\hline
\end{tabular}

Примечание. Результаты цитогенетического анализа: мальчики с СДВГ: 19/47 (40.4\%); мальчики без СДВГ (контр. группа): 1/51 (1.96\%). $X^{2}=22.3$; $\mathrm{P}<0.05 ;$ OR $=33.9 ; 95 \%$ CI 4.31-267.1;

Согласно рассчитанному коэффициенту соотношения шансов риск развития гиперактивного синдрома у пациентов-мальчиков с Үр и Yq+ достоверно выше в 33.9 раза $\left(x^{2}=22.3 ; \mathrm{P}<0.05 ; \mathrm{OR}=33.9\right.$; $95 \% \mathrm{CI}$ 4.31-267.1) по сравнению мальчиков без хромосомной изменении (без Ypss илиYqss+).

Оценка изменения длины $\mathrm{Y}$ хромосомы носила объективносубъективный характер. Изменение относительной длины Y-хромосомы устанавливали на основании ее сравнения с хромосомами 21 и 22 (Gгруппа мелких акроцентрических хромосом) и хромосомой 18 (мелкая субметацентрическая хромосома группы Е). В случае, когда длина Yхромосомы была сопоставима с хромосомой 18 , но в 1,5-2 раза больше длины хромосом группы $\mathrm{G}$, ее считали увеличенной. Если Ү-хромосома была равна по размеру или незначительно больше хромосом группы G, но меньше хромосомы 18, то данный 
Saidkhodjaeva S.N. Cytogenetic manifestations of chromosomal rearrangements in karyotypes of children with attention deficit hyperactivity disorder.

цитогенетический вариант ее длины рассматривался как норма.

A.

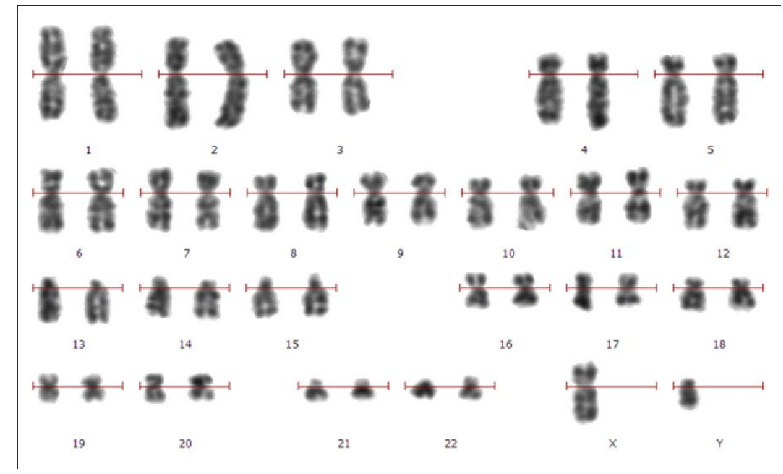

Б.

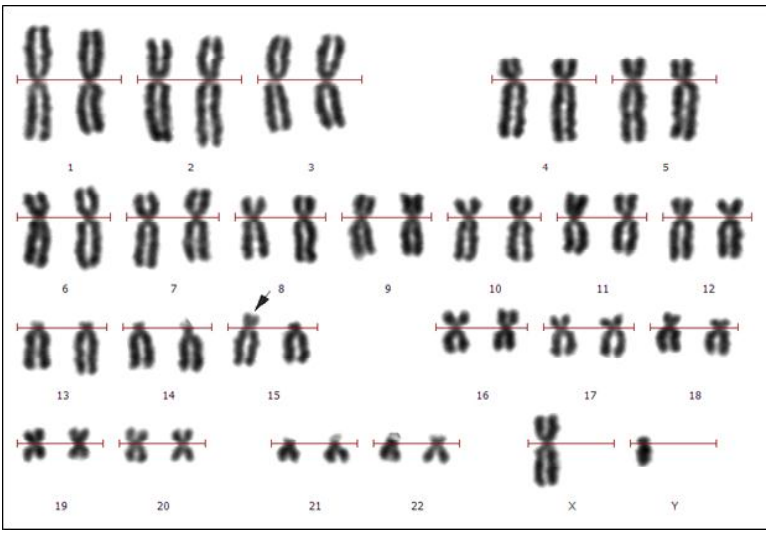

Рис.1 Сравнительная оценка длины Yхромосомы на основании сопоставления с мелкими акроцентрическими хромосомами хромосомами Gгруппы (21 и 22) и мелкой субметоцентрической 18-ой хромосомой из Е-группы.

Изменение размеров затрагивало в основном длинное (q) плечо. Известно, что основной массив гетерохроматина (Gh) в Y-хромосоме, за исключением небольших участков в прицентромерной области (p11.1q11.1), расположен именно в q-плече (локус q12). Изменение размеров qплеча было отмечено как у детей с СДВГ $(38,3 \pm 7,1 \%)$, так и у субъектов контроля $(14,3 \pm 7,6 \%)$. При этом разница при сравнении данных обеих групп была достоверной $(\mathrm{t}=2,31$; $\mathrm{p}<0,05)$, что подтверждало не случайность данного цитогенетического варианта и его связь с развитием СДВГ.

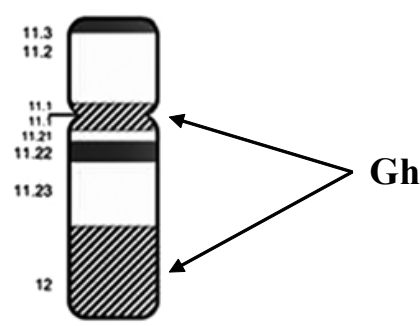

Рис.2 Карта Y-хромосомы с расположением бендов и гетерохроматировых (Gh) участков (обозначены стрелкой) в соответствии с международной системой классификации хромосом ISCN 2009. http://atlasgeneticsoncology.org/ISCN09/ ISCN09.html

В одном случае $(2,1 \pm 2,1 \%)$ нами было отмечено увеличение короткого (p) плеча. Это изменение могло произойти как за счет амплификации региона гетерохроматина в р-плече Yхромосомы (локус р11.1), так и за счет встраивания (инсерции) фрагмента другой хромосомы. В последнем случае перестройка могла затрагивать не только гетерохроматиновую, но и эухроматиновую область. Также не исключен вариант неравномерного транслакационного обмена фрагментами с другими хромосомами. Однако единичность случая увеличения р-плеча в группе больных говорит о недостоверности данного события, его случайности и отсутствии связи с СДВГ в нашем исследовании.

Необходимо отметить, что все выявленные нами случаи увеличения длины Y-хромосомы были полными цитогенетическими вариантами, когда обнаруженные изменения могут быть найдены во всех клетках организма, включая половые. Это говорит о возможности передачи вариантов длины Y-хромосомы, а также 
связанной с ней предрасположенностью к СДВГ потомству, и, следовательно, предполагает наличие наследственного фактора в развитии синдрома. Однако данная версия носит гипотетический характер и нуждается в подтверждении дальнейшими дополнительными исследованиями в расширенных выборках.

\section{Выводы:}

Таким образом, проведенное нами исследование кариотипа показало, что у детей с СДВГ цитогенетически детектируемые изменения встречаются в 33.9 раза чаще, чем у детей без клинических признаков синдрома. Цитогенетических изменений, связанных с количественными перестройками, а также со структурными изменениями аутосом и Х-хромосомы нами не выявлено, однако нами было отмечено достоверное увеличение доли кариотипов с Y-хромосомой, размеры которой превышали норму, установленную на основании сравнения с хромосомами группы $\mathrm{G}$ и хромосомой 18. Было установлено, что изменение длины Ү-хромосомы реализуется за счет увеличения материала ее q-плеча, что согласуется с данными других исследований о влиянии на процесс развития СДВГ эффекта увеличения "дозы" генов. На основании проведенного исследования нами сделан вывод о том, что увеличение длины Y-хромосомы ассоциировано с СДВГ, может быть одним из факторов прогнозирования вероятности развития синдрома и использоваться в скрининговой и пренатальной диагностике.

Таблица 2. Результаты цитогенетического исследования у детей с СДВГ и субъектов контроля

\begin{tabular}{|c|c|c|c|c|c|c|c|c|c|c|c|c|c|c|c|}
\hline \multirow[t]{2}{*}{ Группа } & \multirow[t]{2}{*}{ Пол } & \multirow[t]{2}{*}{$\begin{array}{c}\text { Кол-во } \\
\text { наблю- } \\
\text { дений } \\
\text { (n) }\end{array}$} & \multirow[t]{2}{*}{$\begin{array}{c}\text { Карио- } \\
\text { тип }\end{array}$} & \multicolumn{3}{|c|}{$\begin{array}{c}\text { Кариотип без } \\
\text { цитогенетически } \\
\text { детектируемых } \\
\text { изменений половых } \\
\text { хромосом } \\
\end{array}$} & \multicolumn{3}{|c|}{$\begin{array}{c}\text { Увеличение } \\
\text { Y-хромосомы }\end{array}$} & \multicolumn{3}{|c|}{$\mathrm{Yq}+$} & \multicolumn{3}{|c|}{$\mathrm{Yp}+$} \\
\hline & & & & $\mathrm{n}$ & $\begin{array}{c}\% \\
(\mathrm{M} \pm \mathrm{m})\end{array}$ & $\mathrm{t}, \mathrm{p}$ & $\mathrm{n}$ & $\begin{array}{c}\% \\
(\mathrm{M} \pm \mathrm{m})\end{array}$ & $\mathrm{t}, \mathrm{p}$ & $\mathrm{n}$ & $\begin{array}{c}\% \\
(\mathrm{M} \pm \mathrm{m})\end{array}$ & $\mathrm{t}, \mathrm{p}$ & $\mathrm{n}$ & $\begin{array}{c}\% \\
(\mathrm{M} \pm \mathrm{m})\end{array}$ & $\mathrm{t}, \mathrm{p}$ \\
\hline \multirow{3}{*}{ Основная } & Мальч. & 47 & $46, X Y$ & 28 & $45,1 \pm 7,2$ & $\begin{array}{l}\mathrm{t}=3,88 \\
\mathrm{p}<0,05\end{array}$ & 19 & $40,4 \pm 7,2$ & $\begin{array}{l}\mathrm{t}=2,49 \\
\mathrm{p}<0,05\end{array}$ & 18 & $38,3 \pm 7,1$ & $\begin{array}{l}\mathrm{t}=2,31 \\
\mathrm{p}<0,05\end{array}$ & 1 & $2,1 \pm 2,1$ & $\begin{array}{c}\mathrm{t}=1,0 \\
\mathrm{p}>0,05\end{array}$ \\
\hline & Дев. & 15 & $46, X X$ & 15 & 100 & $\begin{array}{c}\mathrm{t}=0 \\
\mathrm{p}>0,05\end{array}$ & - & - & - & - & - & - & - & - & - \\
\hline & Всего & 62 & $\begin{array}{c}46, \mathrm{XY} \mathrm{и} \\
46, \mathrm{X} \\
\end{array}$ & 43 & $69,3 \pm 5,8$ & $\begin{array}{l}\mathrm{t}=3,49 \\
\mathrm{p}<0,05\end{array}$ & 19 & $30,6 \pm 5,8$ & $\begin{array}{l}\mathrm{t}=3,49 \\
\mathrm{p}<0,05\end{array}$ & 18 & $29,0 \pm 5,8$ & $\begin{array}{l}\mathrm{t}=3,24 \\
\mathrm{p}<0,05\end{array}$ & 1 & $1,6 \pm 1,6$ & $\begin{array}{c}\mathrm{t}=1,0 \\
\mathrm{p}>0,05\end{array}$ \\
\hline \multirow[t]{3}{*}{ Контроль- } & Мальч. & 21 & $46, X Y$ & 18 & $85,7 \pm 7,6$ & - & 1 & $4,8 \pm 7,6$ & - & 1 & $4,8 \pm 7,6$ & - & 0 & 0 & - \\
\hline & Дев. & 24 & $46, \mathrm{XX}$ & 24 & 100 & - & - & - & - & - & - & - & - & - & - \\
\hline & Всего & 45 & $\begin{array}{c}46, \mathrm{XY} \mathrm{и} \\
46, \mathrm{XX}\end{array}$ & 42 & $93,3 \pm 3,7$ & - & 3 & $6,6 \pm 3,7$ & - & 3 & $6,7 \pm 3,7$ & - & 0 & 0 & - \\
\hline
\end{tabular}


Saidkhodjaeva S.N. Cytogenetic manifestations of chromosomal rearrangements in karyotypes of children with attention deficit hyperactivity disorder.

\section{Литература:}

1. Bryant D.M., Hoeft F., Lai S., Lackey J., Roeltgen D., Ross J., Reiss A.L. Sex chromosomes and the brain: a study of neuroanatomy in XYY syndrome // Dev Med Child Neurol. 2012 Dec; 54(12):1149-56. 2. Cordeiro L., Tartaglia N., Roeltge., Rossc J. Social Deficits in Male Children and Adolescents with Sex Chromosome Aneuploidy: A Comparison of XXY, XYY, and XXYY syndromes // Res Dev Disabil. 2012 Jul; 33(4): 1254-1263.

3. Durand Christelle, Kappeler Caroline, Betancur Catalina , et al. Expression and genetic variability of PCDH11Y, a gene specific to Homo sapiens and candidate for susceptibility to psychiatric disorders // Am J Med Genet B Neuropsychiatr Genet. 2006 Jan; 141(1): 67-70.

4. Lepage J.F., Hong D.S., Raman M., Marzelli M., Roeltgen D.P., Lai S., Ross J., Reiss A.L. Brain morphology in children with 47, XYY syndrome: a voxel- and surface-based morphometric study // Genes Brain Behav. 2014 Feb; 13(2):127-34.

5. Loke H., Harley V., Lee J. Biological factors underlying sex differences in neurological disorders // Int J Biochem Cell Biol. 2015 Aug; 65:139-50.

6. McConville B.J., Soudek D., Sroka H., Cot? J., Boag L., Berry J. Length of the Y chromosome and chromosomal variants in inpatient children with psychiatric disorders: two studies // Can J Psychiatry. 1983 Feb; 28(1):8-13.

7. Paoloni-Giacobino A., Lespinasse J. Chromosome Y polysomy: a non-mosaic 49, XYYYY case // Clin Dysmorphol. 2007 Jan; 16(1): 65-66.

8. Ross J.L., Roeltgen D.P., Kushner H., Zinn A.R., Reiss A., Bardsley M.Z., McCauley E., Tartaglia N. Behavioral and social phenotypes in boys with 47, XYY syndrome or 47, XXY Klinefelter syndrome // Pediatrics. 2012 Apr; 129(4):769-78.

9. Mulligan A., Gill M., Fitzgerald M. A case of ADHD and a major Y chromosome abnormality // J Atten Disord. 2008 Jul; 12(1):103-5.

10. Ross J.L., Tartaglia N., Merry D.E., Dalva M., Zinn A.R. Behavioral phenotypes in males with XYY and possible role of increased NLGN4Y expression in autism features // Genes Brain Behav. $2015 \mathrm{Feb} ; 14(2): 137-44$.

11. Tartaglia N., Davis S., Hansen R., Hagerman R. Abstract: attention deficit hyperactivity disorder and autism spectrum disorders in males with XXY, XYY, and XXYY syndromes // J Intel Dis Res. 2006; 50:787.

12. Tartaglia Nicole, Ayari Natalie, Howell Susan, D'Epagnier Cheryl, Zeitler Philip.48, XXYY, 48, XXXY and 49, XXXXY syndromes: not just variants of Klinefelter syndrome // Acta Paediatr. 2011 Jun; 100(6): 851-860.

13. Tartaglia N.R., Ayari N., Hutaff-Lee C., Boada R. Attention-deficit hyperactivity disorder symptoms in children and adolescents with sex chromosome aneuploidy: XXY, XXX, XYY, and XXYY // J Dev Behav Pediatr. 2012 May; 33(4):309-18.

14.Visootsak Jeannie, Graham John M. Social Function in Multiple X and Y Chromosome Disorders: XXY, XYY, XXYY, XXXY // Dev Disabil Res Rev. 2009; 15(4): 328-332. 